Press

\title{
Calendar of Events
}

$6^{\text {th }}$ World Congress of Neurorehabilitation

March 21-25, 2010

Vienna, Austria

Website www.wcnr2010.org

American Academy of Neurology

$62^{\text {nd }}$ Annual Meeting

April 10-17, 2010

Toronto, Ontario, Canada

Website http://am.aan.com/

American Occupational Therapy Association

$90^{\text {th }}$ Annual Conference and Expo

April 29-May 2, 2010

Orlando, FL

Website http://www.aota.org/ConfandEvents/ 2010AnnualConference.aspx

International Child Neurology Association

$11^{\text {th }}$ International Child Neurology Congress

May 2-7, 2010

Cairo, Egypt

Website http://www.icnc2010.com/

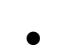

The Pediatric Orthopedic Society of North America

May 3-7, 2010

Waikaloa, HI

Website http://www.posna.org/
European Society of Movement Analysis in Adults and Children (ESMAC)

$19^{\text {th }}$ Annual General Meeting

May 10-14, 2010

Miami, FL

Website http://www.esmac.org/

$22^{\text {nd }}$ Annual Meeting of the European Academy of Childhood Disability

May 27-29, 2010

Brussels, Belgium

Website http://www.eacd2010.com

American Urological Association

May 29-June 3, 2010

San Francisco, CA

Website http://www.auanet.org

American Physical Therapy Association Annual Conference

June 16-19, 2010

Boston, MA

Website http://www.apta.org

Society for Research into Hydrocephalus and Spina Bifida

$54^{\text {th }}$ Scientific Meeting

July 7-10, 2010

University of British Columbia

Vancouver, Canada

Website http://srhsb.org/ 